\title{
STABILITY PROPERTIES IN NONLINEAR DISCRETE VOLTERRA EQUATIONS WITH UNBOUNDED DELAY
}

\author{
Sung Kyu Choi*, Yunhee Kim**, Namjip Koo***, and Chanmi \\ $\mathrm{YUN} * * * *$
}

\begin{abstract}
We study some stability properties in discrete Volterra equations by employing to change Yoshizawa's results in [13] for the nonlinear equations into results for the nonlinear discrete Volterra equations with unbounded delay.
\end{abstract}

\section{Introduction}

Consider the discrete Volterra equation with unbounded delay

$$
x(n+1)=f(n, x(n))+\sum_{j=-\infty}^{n} B(n, j, x(j), x(n)), n \in \mathbb{Z}^{+},
$$

where $f: \mathbb{Z} \times \mathbb{R}^{d} \rightarrow \mathbb{R}^{d}$ is continuous in $x \in \mathbb{R}^{d}$ for every $n \in \mathbb{Z}$, and $B: \mathbb{Z} \times \mathbb{Z} \times \mathbb{R}^{d} \times \mathbb{R}^{d} \rightarrow \mathbb{R}^{d}$ is continuous in $x, y \in \mathbb{R}^{d}$ for every $j, n \in$ $\mathbb{Z}, j \leq n$. Eq. (1.1) is a discrete analogue of the integro-differential equation

$$
x^{\prime}(t)=\hat{f}(t, x(t))+\int_{-\infty}^{0} F(t, s, x(t+s), x(t)) d s,
$$

where $\hat{f}: \mathbb{R} \times \mathbb{R}^{n} \rightarrow \mathbb{R}^{n}$ is continuous and $F: \mathbb{R} \times \mathbb{R}^{-} \times \mathbb{R}^{n} \times \mathbb{R}^{n} \rightarrow \mathbb{R}^{n}$ is continuous.

In [5], Hamaya studied the relationship between total stability and stability under disturbances from hull for Eq. (1.2).

Received December 12, 2012; Accepted January 11, 2013.

2010 Mathematics Subject Classification: Primary 39A10, 39A24, 39A30.

Key words and phrases: discrete Volterra equation with unbounded delay, BS-stability, $(K, \rho)$-stability, $\left((K, \rho), \mathbb{R}^{d}\right)$-stability, total stability, almost periodic solution.

This work was supported by Basic Science Research Program through the National Research Foundation of Korea(NRF) funded by the Ministry of Education, Science and Technology(NRF-2010-0008835). 
Song and Tian [10] investigated the existence of periodic and almost periodic solutions for Eq. (1.1) by means of $(K, \rho)$-stability conditions. Their results are to extend results of Hamaya [4] to discrete Volterra equations. Also, Song [11] proved that if the bounded solution of (1.1) is uniformly asymptotically stable, then (1.1) has an almost periodic solution.

Choi and Koo [2] investigated the existence of an almost periodic solution of (1.1) as a discretization of the Hamaya's results in [5]. Also, Choi et al. [3] studied the total stability for the discrete Volterra equation

$$
x(n+1)=f(n, x(n))+\sum_{j=-\infty}^{0} B(n, j, x(n+j), x(n))+h\left(n, x_{n}\right) .
$$

Sell [9] introduced the concept of weak uniform asymptotic stability to obtain the existence theorem for almost periodic solutions in ordinary differential equations. In [12], Xia and Cheng studied the existence of almost periodic solutions for difference equation by using the concept of global quasi-uniform asymptotic stability.

Hamaya [5] showed that two concepts of $(K, \rho)$-weak uniform asymptotic stability and $(K, \rho)$-uniform asymptotic stability for $(1.1)$ are equivalent. Moreover, he obtained the existence of almost periodic solutions in (1.1) by using $(K, \rho)$-weak uniform asymptotic stability.

The space $B C$ which consists of all bounded functions on $\mathbb{R}^{-}=$ $(-\infty, 0]$ is one of the important classes for the space of initial functions in the theory of functional differential equations with unbounded delay as well as integrodifferential equations. In connection with the stability problems, there are two ways to provide the metric structure in $B C$. One way is to provide it with the supremum norm, and the other is of compact open topology induced by the $\rho$-metric. So there are two stability concepts referred to as the $B C$-stabilities and the $\rho$-stabilities, respectively. In [8], Murakami and Yoshizawa investigated the relationships between $B C$-stabilities and $\rho$-stabilities in functional differential equations with unbounded delay.

In this paper, we study some stability properties for (1.1) by employing to change Yoshizawa's results in [13] for the nonlinear equations into results for the discrete Volterra equations with unbounded delay. Also, we investigate the $B S$-s.d.(stability under disturbance) and $\rho$-s.d. for (1.1). 


\section{Preliminaries}

In what follows, we denote by $\mathbb{R}, \mathbb{R}^{+}, \mathbb{R}^{-}$, respectively, the set of real numbers, the set of nonnegative real numbers, and the set of nonpositive real numbers. Also, we denote by $\mathbb{Z}, \mathbb{Z}^{+}, \mathbb{Z}^{-}$, the set of integers, the set of nonnegative integers, and the set of nonpositive integers, respectively. Let $\mathbb{R}^{d}$ denote $d$-dimensional Euclidean space with a norm $|\cdot|$.

Definition 2.1. A continuous function $\hat{f}: \mathbb{R} \times \mathbb{R}^{n} \rightarrow \mathbb{R}^{n}$ is said to be almost periodic in $t \in \mathbb{R}$ uniformly for $x \in \mathbb{R}^{n}$ if for any $\varepsilon>0$ there corresponds a number $l=l(\varepsilon)>0$ such that any interval of length $l$ contains a number $\tau$ for which

$$
|\hat{f}(t+\tau, x)-\hat{f}(t, x)|<\varepsilon
$$

for all $t \in \mathbb{R}$ and $x \in \mathbb{R}^{n}$.

Let $F(t, s, x, y)$ be a function which is defined and continuous for $t \in \mathbb{R}$ and $(s, x, y) \in R^{*}=\mathbb{R}^{-} \times \mathbb{R}^{n} \times \mathbb{R}^{n}$.

Definition 2.2. $F(t, s, x, y)$ is said to be almost periodic in $t$ uniformly for $(s, x, y) \in R^{*}$ if for any $\varepsilon>0$ and any compact set $K^{*} \subset R^{*}$, there exists an $L=L\left(\varepsilon, K^{*}\right)>0$ such that any interval of length $L$ contains a $\tau$ for which

$$
|F(t+\tau, s, x, y)-F(t, s, x, y)|<\varepsilon
$$

for all $t \in \mathbb{R}$ and all $(s, x, y) \in K^{*}$.

Definition 2.3. A continuous function $f: \mathbb{Z} \times \mathbb{R}^{d} \rightarrow \mathbb{R}^{d}$ is said to be almost periodic in $n \in \mathbb{Z}$ uniformly for $x \in \mathbb{R}^{d}$ if for every $\varepsilon>0$ and every compact set $K \subset \mathbb{R}^{d}$, there corresponds an integer $N=N(\varepsilon, K)>0$ such that among $N$ consecutive integers there is one, here denoted by $p$, such that

$$
|f(n+p, x)-f(n, x)|<\varepsilon
$$

for all $n \in \mathbb{Z}$, uniformly for $x \in \mathbb{R}^{d}$.

Definition 2.4. A set $\Sigma \subset Z^{*}=\mathbb{Z}^{-} \times \mathbb{R}^{d} \times \mathbb{R}^{d}$ is said to be compact if there exists a finite integer set $\Delta \subset \mathbb{Z}^{-}$and compact set $\Theta \subset \mathbb{R}^{d} \times \mathbb{R}^{d}$ such that $\Sigma=\Delta \times \Theta$.

Definition 2.5. Let $B: \mathbb{Z} \times \mathbb{Z} \times \mathbb{R}^{d} \times \mathbb{R}^{d} \rightarrow \mathbb{R}^{d}$ be continuous for $x, y \in \mathbb{R}^{d}$, for any $j, n \in \mathbb{Z}, j \leq n . B(n, j, x, y)$ is said to be almost periodic in $n$ uniformly for $(j, x, y) \in Z^{*}$ if for any $\varepsilon>0$ and any 
compact set $K^{*} \subset Z^{*}$, there exists a number $l=l\left(\varepsilon, K^{*}\right)>0$ such that any discrete interval of length $l$ contains a $\tau$ for which

$$
|B(n+\tau, j, x, y)-B(n, j, x, y)|<\varepsilon
$$

for all $n \in \mathbb{Z}$ and all $(j, x, y) \in K^{*}$.

For the basic results of almost periodic functions, see [14].

Let $B S=B S\left(\mathbb{Z}^{-}, \mathbb{R}^{d}\right)$ denote the space of all $\mathbb{R}^{d}$-valued bounded functions on $\mathbb{Z}^{-}$with

$$
|\phi|_{B S}=\sup _{n \in \mathbb{Z}^{-}}|\phi(n)|<\infty, \phi \in B S .
$$

Let $x:\{n \in \mathbb{Z}: n \leq k\} \rightarrow \mathbb{R}^{d}$ for any integer $k$. For any $n \leq k$, we define $x_{n}: \mathbb{Z}^{-} \rightarrow \mathbb{R}^{d}$ by

$$
x_{n}(j)=x(n+j), j \leq 0 .
$$

Consider the discrete Volterra equation with unbounded delay

$$
\begin{aligned}
x(n+1) & =f(n, x(n))+\sum_{j=-\infty}^{n} B(n, j, x(j), x(n)), n \in \mathbb{Z}^{+}, \\
& =f(n, x(n))+\sum_{j=-\infty}^{0} B(n, n+j, x(n+j), x(n)),
\end{aligned}
$$

where $f: \mathbb{Z} \times \mathbb{R}^{d} \rightarrow \mathbb{R}^{d}$ is continuous in $x \in \mathbb{R}^{d}$ for every $n \in \mathbb{Z}$ and is almost periodic in $n \in \mathbb{Z}$ uniformly for $x \in \mathbb{R}^{d}, B: \mathbb{Z} \times \mathbb{Z}^{-} \times \mathbb{R}^{d} \times \mathbb{R}^{d} \rightarrow \mathbb{R}^{d}$ is continuous in $x, y \in \mathbb{R}^{d}$ for any $j \leq n \in \mathbb{Z}$ and is almost periodic in $n$ uniformly for $(j, x, y) \in Z^{*}$. We assume that, given $\phi \in B S\left(\mathbb{Z}^{-}, \mathbb{R}^{d}\right)$, there is a solution $x$ of (2.1) such that $x(n)=\phi(n)$ for $n \in \mathbb{Z}^{-}$, passing through $(0, \phi)$. Denote by this solution $x(n)=x(n, \phi)$.

Let $K$ be any compact subset of $\mathbb{R}^{d}$ such that $\phi(j) \in K$ for all $j \leq 0$ and $x(n)=x(n, \phi) \in K$ for all $n \geq 1$.

For any $\phi, \psi \in B S\left(\mathbb{Z}^{-}, \mathbb{R}^{d}\right)$, we set

$$
\rho(\phi, \psi)=\sum_{q=0}^{\infty} \frac{\rho_{q}(\phi, \psi)}{2^{q}\left[1+\rho_{q}(\phi, \psi)\right]},
$$

where $\rho_{q}(\phi, \psi)=\max _{-q \leq m \leq 0}|\phi(m)-\psi(m)|, q \geq 0$. Then $\rho$ defines a metric on the space $B S\left(\mathbb{Z}^{-}, \mathbb{R}^{d}\right)$. Note that the induced topology by $\rho$ is the same as the topology of convergence on any finite subset of $\mathbb{Z}^{-}[8]$. 
In view of almost periodicity, for any sequence $\left(n_{k}^{\prime}\right) \subset \mathbb{Z}^{+}$with $n_{k}^{\prime} \rightarrow$ $\infty$ as $k \rightarrow \infty$, there exists a subsequence $\left(n_{k}\right) \subset\left(n_{k}^{\prime}\right)$ such that

$$
f\left(n+n_{k}, x\right) \rightarrow g(n, x)
$$

uniformly on $\mathbb{Z} \times S$ for any compact set $S \subset \mathbb{R}^{d}$,

$$
B\left(n+n_{k}, n+l+n_{k}, x, y\right) \rightarrow D(n, n+l, x, y)
$$

uniformly on $\mathbb{Z} \times S^{*}$ for any compact set $S^{*} \subset Z^{*}, g(n, x)$ and $D(n, n+$ $l, x, y)$ are also almost periodic in $n$ uniformly for $x \in \mathbb{R}^{d}$, and almost periodic in $n$ uniformly for $(j, x, y) \in Z^{*}$, respectively. We define

$$
\begin{aligned}
\Omega(f, B)= & \{(g, D):(2.3) \text { and }(2.4) \text { hold for some sequence } \\
& \left.\left(n_{k}\right) \subset \mathbb{Z}^{+} \text {with } n_{k} \rightarrow \infty \text { as } k \rightarrow \infty\right\} .
\end{aligned}
$$

Note that $(f, B) \in \Omega(f, B)$ and for any $(g, D) \in \Omega(f, B)$, we can assume the almost periodicity of $g$ and $D$, respectively [8].

Definition 2.6. If $(g, D) \in \Omega(f, B)$, then the equation

$$
x(n+1)=g(n, x(n))+\sum_{j=-\infty}^{n} D(n, j, x(j), x(n)), n \in \mathbb{Z}^{+}
$$

is called the limiting equation of (2.1).

For the compact set $K$ in $\mathbb{R}^{d},(p, P) \in \Omega(f, B),(q, Q) \in H(f, B)$, we define $\pi(p, q)$ and $\pi(P, Q)$ by

$$
\begin{aligned}
\pi(p, q) & =\sup \{|p(n, x)-q(n, x)|: n \in \mathbb{Z}, x \in K\} \\
\pi(P, Q) & =\sum_{N=1}^{\infty} \frac{\pi_{N}(P, Q)}{2^{N}\left[1+\pi_{N}(P, Q)\right]}
\end{aligned}
$$

where

$$
\begin{array}{r}
\pi_{N}(P, Q)=\sup \{|P(n, j, x, y)-Q(n, j, x, y)|: \\
n \in \mathbb{Z}, j \in[-N, 0], x, y \in K\},
\end{array}
$$

and

$$
\pi((p, P),(q, Q))=\max \{\pi(p, q), \pi(P, Q)\},
$$

respectively. This definition is a discrete analogue of Hamaya's definition in $[5]$.

We recall the definitions of various stabilities in [7].

Definition 2.7. The bounded solution $u(n)$ of Eq. (1.1) is said to be 
(i) $(K, \rho)$-stable (in short, $(K, \rho)$-S) if for any $\varepsilon>0$ there exists a $\delta=\delta\left(n_{0}, \varepsilon\right)>0$ such that if $n_{0} \geq 0$ and $\rho\left(x_{n_{0}}, u_{n_{0}}\right)<\delta$, then

$$
\rho\left(x_{n}, u_{n}\right)<\varepsilon, n \geq n_{0},
$$

where $x(n)$ is a solution of (1.1) through $\left(n_{0}, \phi\right)$ such that $x_{n_{0}}(s)=$ $\phi(s) \in K$ for all $s \leq 0$.

(ii) $(K, \rho)$-uniformly stable $((K, \rho)$-US) if for any $\varepsilon>0$ there exists a $\delta=\delta(\varepsilon)>0$ such that if $n_{0} \geq 0$ and $\rho\left(x_{n_{0}}, u_{n_{0}}\right)<\delta$, then

$$
\rho\left(x_{n}, u_{n}\right)<\varepsilon, n \geq n_{0},
$$

where $x(n)$ is a solution of (1.1) through $\left(n_{0}, \phi\right)$ such that $x_{n_{0}}(s)=$ $\phi(s) \in K$ for all $s \leq 0$.

(iii) $(K, \rho)$-equi asymptotically stable $\left((K, \rho)-E_{q} \mathrm{AS}\right)$ if it is $(K, \rho)-\mathrm{S}$ and for any $\varepsilon>0$ there exists a $\delta_{0}=\delta_{0}\left(n_{0}\right)>0$ and a $T=T\left(n_{0}, \varepsilon\right)>0$ such that if $n_{0} \geq 0$ and $\rho\left(x_{n_{0}}, u_{n_{0}}\right)<\delta_{0}$, then

$$
\rho\left(x_{n}, u_{n}\right)<\varepsilon, n \geq n_{0}+T,
$$

where $x(n)$ is a solution of $(1.1)$ through $\left(n_{0}, \phi\right)$ such that $x_{n_{0}}(s)=$ $\phi(s) \in K$ for all $s \leq 0$.

(iv) $(K, \rho)$-weakly uniformly asymptotically stable $((K, \rho)$-WUAS) if it is $(K, \rho)$-US and there exists a $\delta_{0}>0$ such that if $n_{0} \geq 0$ and $\rho\left(x_{n_{0}}, u_{n_{0}}\right)<\delta_{0}$, then

$$
\rho\left(x_{n}, u_{n}\right) \rightarrow 0 \text { as } n \rightarrow \infty,
$$

where $x(n)$ is a solution of (1.1) through $\left(n_{0}, \phi\right)$ such that $x_{n_{0}}(s)=$ $\phi(s) \in K, s \leq 0$.

(v) $(K, \rho)$-uniformly asymptotically stable $((K, \rho)$-UAS $)$ if it is $(K, \rho)$ US and if the $\delta_{0}$ and the $T$ in the above (iii) are of independent of $n_{0}$.

Definition 2.8. The bounded solution $x(n)$ of $(2.1)$ is said to be $B S$ - stable under disturbances from $\Omega(f, B)$ with respect to $K$ (in short, $B S$-s.d. $\Omega(f, B))$ if for any $\varepsilon>0$, there exists an $\eta=\eta(\varepsilon)>0$ such that if $(g, D) \in \Omega(f, B)$ with $\pi((f, B),(g, D))<\eta$ and $\left|x_{n_{0}}-u_{n_{0}}\right|_{B S}<\eta$ for some $n_{0} \geq 0$, then

$$
\left|x_{n}-u_{n}\right|_{B S}<\varepsilon, n \geq n_{0},
$$

where $x(n)$ is a solution of the limiting equation (2.6), which passes through $\left(n_{0}, \phi\right)$ such that $x_{n_{0}}(j)=\phi(j) \in K, j \leq 0$.

Definition 2.9. The bounded solution $x(n)$ of $(2.1)$ is said to be $(K, \rho)$-stable under disturbances from $\Omega(f, B)$ (in short, $(K, \rho)$-s.d. $\Omega(f, B)$ ) 
if for any $\varepsilon>0$, there exists an $\eta=\eta(\varepsilon)>0$ such that if $(g, D) \in \Omega(f, B)$ with $\pi((f, B),(g, D))<\eta$ and $\rho\left(x_{n_{0}}, u_{n_{0}}\right)<\eta$ for some $n_{0} \geq 0$, then

$$
\rho\left(x_{n}, u_{n}\right)<\varepsilon, n \geq n_{0},
$$

where $x(n)$ is a solution of the limiting equation (2.6), which passes through $\left(n_{0}, \phi\right)$ such that $x_{n_{0}}(j)=\phi(j) \in K, j \leq 0$.

Definition 2.10. The bounded solution $x(n)$ of $(2.1)$ is said to be $\left((K, \rho), \mathbb{R}^{d}\right)$-stable under disturbances from $\Omega(f, B)$ (in short, $\left((K, \rho), \mathbb{R}^{d}\right)$ s.d. $\Omega(f, B))$ if for any $\varepsilon>0$, there exists an $\eta=\eta(\varepsilon)>0$ such that if $(g, D) \in \Omega(f, B)$ with $\pi((f, B),(g, D))<\eta$ and $\left|x_{n_{0}}-u_{n_{0}}\right|<\eta$ for some $n_{0} \geq 0$, then

$$
|x(n)-u(n)|<\varepsilon, n \geq n_{0},
$$

where $x(n)$ is a solution of the limiting equation (2.6), which passes through $\left(n_{0}, \phi\right)$ such that $x_{n_{0}}(j)=\phi(j) \in K, j \leq 0$.

Remark 2.11. The $(K, \rho)$-s.d. $\Omega(f, B)$ implies the $B S$-s.d. $\Omega(f, B)$ because of $\rho(\phi, \psi) \leq|\phi-\psi|_{B S}$ for $\phi, \psi \in B S\left(\mathbb{Z}^{-}, \mathbb{R}^{d}\right)$.

\section{Main results}

We consider the discrete Volterra equations with unbounded delay

$$
x(n+1)=f(n, x(n))+\sum_{j=-\infty}^{0} B(n, j, x(j), x(n)),
$$

where $f: \mathbb{Z} \times \mathbb{R}^{d} \rightarrow \mathbb{R}^{d}$ is continuous at second variable $x \in \mathbb{R}^{d}$ with $f(n, 0)=0$ and $B: \mathbb{Z} \times \mathbb{Z}^{-} \times \mathbb{R}^{d} \times \mathbb{R}^{d} \rightarrow \mathbb{R}^{d}$ is continuous for $x, y \in \mathbb{R}^{d}$. We denote by $x(n)=x\left(n, n_{0}, x_{0}\right)$ a solution of (3.1) through $\left(n_{0}, x_{0}\right)$.

TheOREM 3.1. Let $u(n)$ be a bounded solution of Eq. (3.1), that is, for some $M>0,|u(n)| \leq M, n \geq 0$. If $u(n)$ is $(K, \rho)$-WUAS, then it is $(K, \rho)-E_{q} A S$.

Proof. Since $u(n)$ is $(K, \rho)$-US, it is $(K, \rho)$-S.

We show that for any $\varepsilon>0$ there exists a $T=T\left(n_{0}, \varepsilon\right)>0$ such that if $n_{0} \geq 0$ and $\rho\left(x_{n_{0}}, u_{n_{0}}\right)<\delta_{0}$, then

$$
\rho\left(x_{n}, u_{n}\right)<\varepsilon, n \geq n_{0}+T,
$$

where $x(n)$ is a solution of (3.1) through $\left(n_{0}, \phi\right)$ such that $x_{n_{0}}(s)=$ $\phi(s), s \leq 0$. Suppose that there does not exist such $T$. Then there exist 
an $\varepsilon>0$, an $n_{0} \geq 0$, and sequences $\left(x^{k}\right)$ with $x^{k}=x\left(n_{k}, n_{0}, x_{k}\right),\left(n_{k}\right)$ with $n_{k} \rightarrow \infty$ as $k \rightarrow \infty$ such that

$$
\rho\left(x_{n_{0}}^{k}, u_{n_{0}}\right)<\delta_{0}
$$

and

$$
\begin{aligned}
\rho\left(u_{n_{k}}, x_{n_{k}}^{k}\right) & \geq \varepsilon, \\
\rho\left(u_{n}, x_{n}^{k}\right) & <\varepsilon, n \in\left[n_{1}, n_{k}\right),
\end{aligned}
$$

where $x^{k}(n)$ is a solution of (3.1) such that $x_{n_{k}}^{k}(s) \in K$ for $s \leq 0$ and $x^{k}(n) \in K$ on $\mathbb{Z}^{+}$. Note that the sequence $\left(x^{k}(n)\right)$ is uniformly bounded. Then there exists a solution $\bar{x}(n)$ of (3.1) defined for all $n \geq n_{0}$ such that

$$
\rho\left(u_{n_{0}}, \bar{x}_{n}\right) \leq \delta_{0} .
$$

Moreover, a subsequence of $\left(x^{k}(n)\right)$ tends to $\bar{x}(n)$ uniformly on any compact interval. Since every solution tends to $u(n)$ as $n \rightarrow \infty$, there exists an $n_{1}$ such that

$$
\rho\left(u_{n_{1}}, \bar{x}_{n_{1}}\right)<\frac{1}{2} \delta(\varepsilon),
$$

where $\delta(\cdot)$ is the number given in $(K, \rho)$-US. We denote by $\left(x^{k}(n)\right)$ the subsequence again. Then we have

$$
\rho\left(\bar{x}_{n_{1}}, x_{n_{1}}^{k}\right)<\frac{1}{2} \delta(\varepsilon)
$$

for sufficiently large $k$. From (3.3) and (3.4), we obtain

$$
\rho\left(x_{n_{1}}^{k}, u_{n_{1}}\right)<\delta(\varepsilon) \text {. }
$$

By the uniform stability of $u(n)$, we have

$$
\rho\left(x_{n}^{k}, u_{n}\right)<\varepsilon, n \geq n_{1},
$$

which contradicts (3.2). This completes the proof.

Theorem 3.2. Suppose that $f(n, x)$ in (3.1) is $\omega$-periodic in $n$. We assume the zero solution of Eq. (3.1). If the bounded solution $u(n)$ of (3.1) is $(K, \rho)$-WUAS, then it is $(K, \rho)-U A S$.

Proof. Assume that there exists an $M>0$ such that $|u(n)| \leq M$. Since $u(n)$ is $(K, \rho)$-US, there exists a $\delta_{0}^{*}>0$ such that if $n_{0} \geq 0$ and $\rho\left(x_{n_{0}}, u_{n_{0}}\right)<\delta_{0}^{*}$, then

$$
\rho\left(x_{n}, u_{n}\right)<\frac{\delta_{0}}{2}, n \geq n_{0},
$$


where $\delta_{0}$ is the number for $(K, \rho)$-WUAS of $u(n)$ and $x(n)$ is a solution of (3.1) through $\left(n_{0}, \phi\right)$ such that $x_{n_{0}}(s)=\phi(s) \in K$ for $s \leq 0$.

Suppose that $u(n)$ is not $(K, \rho)$-UAS. Then there exist an $\varepsilon>0$, and sequences $\left(k_{j}\right) \subset \mathbb{Z}^{+}$with $k_{j} \rightarrow \infty$ as $j \rightarrow \infty,\left(x^{k_{j}}\right)$ with $x^{k_{j}}(n)=$ $x\left(n, k_{j}, \bar{x}_{k_{j}}\right),\left(\tau_{k_{j}}\right)$ with $\tau_{k_{j}} \rightarrow \infty$ as $j \rightarrow \infty$ such that

$$
\begin{aligned}
& \rho\left(x^{k_{j}}, u_{k_{j} \omega}\right)<\frac{\delta_{0}}{2} \\
& \rho\left(x_{\tau_{k_{j}}}^{k_{j}}, u_{k_{j} \omega+\tau_{k_{j}}}\right) \geq \varepsilon .
\end{aligned}
$$

Since $\left|u\left(k_{j} \omega\right)\right| \leq M$, there exist a sequence $\left(m_{j}\right) \subset\left(k_{j}\right)$ with $m_{j} \rightarrow \infty$ as $j \rightarrow \infty$ and a function $u_{0}$ such that $u_{m_{j} \omega} \rightarrow u_{0}$. Then there exists an integer $p>0$ such that

$$
\rho\left(u_{m_{j} \omega}, u_{0}\right)<\frac{\delta_{0}}{4}, j \geq p .
$$

Thus we obtain

$$
\rho\left(u_{m_{j} \omega}, u_{m_{p} \omega}\right)<\frac{\delta_{0}}{2}, j \geq p .
$$

Form (3.5) with $k_{j}=m_{j}$ and (3.7), we have

$$
\rho\left(x^{m_{j}}, u_{m_{p} \omega}\right)<\delta_{0} .
$$

In view of Theorem 3.1, there exists a $T=T\left(m_{p} \omega, \frac{\varepsilon}{2}\right)>0$ such that

$$
\rho\left(\tilde{x}_{n}, u_{n}\right)<\frac{\varepsilon}{2}, n \geq m_{p} \omega+T
$$

and

$$
\rho\left(\tilde{\tilde{x}}_{n}, u_{n}\right)<\frac{\varepsilon}{2}, n \geq m_{p} \omega+T,
$$

where $\tilde{x}(n)=x\left(n, m_{p} \omega, u\left(m_{j} \omega\right)\right)$ and $\tilde{\tilde{x}}(n)=x\left(n, m_{p} \omega, \bar{x}_{m_{j}}\right)$. Then it follows that

$$
\rho\left(\tilde{\tilde{x}}_{n}, \tilde{x}_{n}\right)<\varepsilon, n \geq m_{p} \omega+T .
$$

By (3.9), we have

$$
\rho\left(x^{m_{j}}, u_{n}\right)<\varepsilon, n \geq m_{j} \omega+T .
$$

This contradicts (3.6), because $T=T\left(m_{p} \omega, \frac{\varepsilon}{2}\right)$ depends only on $\varepsilon$. This proves the theorem.

Theorem 3.3. Suppose that $f(n, x)$ in (3.1) is almost periodic in $n$ uniformly for $x \in \mathbb{R}^{d}$. If the solution $u(n)$ of $(3.1)$ is $(K, \rho)$-WUAS, then it is $(K, \rho)-U A S$. 
Proof. Note that $u(n)$ is $(K, \rho)$-US. Then there exists a $\delta_{0}=\delta\left(\delta_{0}\right)>0$ such that if $\rho\left(x_{n_{0}}, u_{n_{0}}\right)<\delta$, then

$$
\rho\left(x_{n}, u_{n}\right)<\delta_{0}, n \geq n_{0} \geq 0,
$$

where $\delta_{0}$ is the number given in $(K, \rho)$-WUAS and $x(n)$ is a solution of (3.1) through $\left(n_{0}, \phi\right)$ such that $x_{n_{0}}(s)=\phi(s) \in K$ for $s \leq 0$.

We show that for $\varepsilon>0$ there exists a $T=T(\varepsilon)>0$ such that if $\rho\left(x_{n_{0}}, u_{n_{0}}\right)<\delta\left(\delta_{0}\right)$ for $n_{0} \geq 0$, then

$$
\rho\left(x_{n_{1}}, u_{n}\right)<\delta(\varepsilon), n_{0} \leq n_{1} \leq n_{0}+T
$$

for some $n_{1}$, where $\delta(\cdot)$ is the number for $(K, \rho)$-US of $u(n)$. Then we obtain

$$
\rho\left(x_{n}, u_{n}\right)<\varepsilon, n \geq n_{0}+T,
$$

that is, $u(n)$ is $(K, \rho)$-UAS. Suppose that there is no such $T$. Then for any $k \geq 1$, there exist sequences $\left(x^{k}\right)$ with $x^{k}(n)=x\left(n, k, \bar{x}_{k}\right)$ and $\left(n_{k}\right) \subset \mathbb{Z}^{+}$such that

$$
\rho\left(x_{n_{0}}^{k}, u_{n_{0}}\right)<\delta\left(\delta_{0}\right)
$$

and

$$
\rho\left(x_{n_{k}}^{k}, u_{n_{k}}\right) \geq \delta(\varepsilon), n_{k} \leq n \leq n_{k}+k .
$$

Let $y^{k}(n)=x\left(n+n_{k}, n_{k}, \bar{x}_{k}\right)$. Then $y^{k}(n)$ is a solution of

$$
\begin{aligned}
x\left(n+n_{k}+1\right) & =f\left(n+n_{k}, x\left(n+n_{k}\right)\right) \\
& +\sum_{j=-\infty}^{0} B\left(n+n_{k}, j, x(j), x\left(n+n_{k}\right)\right),
\end{aligned}
$$

through $\left(0, \bar{x}_{k}\right)$ and

$$
\rho\left(y_{n}^{k}, u_{n}\right)<\delta(\varepsilon), 0 \leq n \leq k .
$$

Note that

$$
\rho\left(x_{n_{0}}^{k}, u_{n_{0}}\right)<\delta\left(\delta_{0}\right) .
$$

Since $f(n, x)$ is almost periodic in $n$ uniformly for $x \in \mathbb{R}^{d}$, there exist $x^{0}, g(n, x), z(n),\left(k_{j}\right) \subset \mathbb{Z}^{+}$such that

$$
\begin{aligned}
& x^{k_{j}} \rightarrow x^{0}, \\
& f\left(n+n_{k_{j}}, x\right) \rightarrow g(n, x)
\end{aligned}
$$

uniformly on $\mathbb{Z}^{+} \times K$, and

$$
y^{k_{j}}(n) \rightarrow z(n)
$$


uniformly on any compact interval on $\mathbb{Z}^{+}$, where $z(n)$ is a solution of the limiting equation

$$
x(n+1)=g(n, x(n))+\sum_{j=-\infty}^{0} D(n, j, x(j), x(n)),(g, D) \in \Omega(f, B)
$$

through $\left(0, x^{0}\right)$. For any $n \geq 0$, there exists a $j$ sufficiently large so that

$$
\rho\left(u_{n}, y_{n}^{k_{j}}\right)+\rho\left(y_{n}^{k_{j}}, z_{n}\right) \leq \rho\left(u_{n}, z_{n}\right) .
$$

Note that for large $j$,

$$
\rho\left(y_{n}^{k_{j}}, u_{n}\right) \geq \delta(\varepsilon)
$$

and

$$
\rho\left(y_{n}^{k_{j}}, z_{n}\right)<\frac{\delta(\varepsilon)}{2}
$$

Thus we have

$$
\rho\left(u_{n}, z_{n}\right) \geq \frac{\delta(\varepsilon)}{2}, n \geq 0
$$

Clearly,

$$
\rho\left(u_{n}, z_{n}\right) \leq \delta_{0}, n \geq 0 .
$$

Since $(f, B) \in \Omega(g, D)$, there exists a sequence $\left(\tau_{k}\right)$ with $\tau_{k} \rightarrow \infty$ as $k \rightarrow \infty$ such that

$$
g\left(n+\tau_{k}, x\right) \rightarrow f(n, x)
$$

uniformly for $n \in \mathbb{Z}$ and $x \in S=\left\{x \in \mathbb{R}^{d}:|x| \leq \delta_{0}\right\}$ as $k \rightarrow \infty$. We set $\eta^{k}(n)=z\left(n+\tau_{k}\right)$. Then $\eta^{k}(n)$ is a solution of

$$
x\left(n+\tau_{k}+1\right)=g\left(n+\tau_{k}, x\right)+\sum_{j=-\infty}^{0} D\left(n+\tau_{k}, j, x(j), x\left(n+\tau_{k}\right)\right)
$$

through $\left(0, z\left(\tau_{k}\right)\right)$, and $\left(\eta^{k}(n)\right)$ is uniformly bounded by (3.13). Therefore there exists a subsequence $\left(\tau_{k_{j}}\right) \subset\left(\tau_{k}\right)$ such that

$$
g\left(n+\tau_{k_{j}}, x\right) \rightarrow f(n, x)
$$

uniformly for $n \in \mathbb{Z}$ and $x \in S$, and

$$
\eta^{k_{j}}(n) \rightarrow \xi(n)
$$


on any compact interval of $\mathbb{Z}^{+}$, where $\xi(n)$ is a solution of (3.1). Then for large $j$, we have

$$
\begin{aligned}
\rho\left(\xi_{n}, u_{n}\right) & \geq \rho\left(\eta_{n}^{k_{j}}, u_{n}\right)+\rho\left(\eta_{n}^{k_{j}}, \xi_{n}\right) \\
& \geq \frac{\delta(\varepsilon)}{2}+\frac{\delta(\varepsilon)}{4}=\frac{3 \delta(\varepsilon)}{4}
\end{aligned}
$$

because $\tau_{k_{j}}>0$ for $j$ sufficiently large and

$$
\rho\left(\eta_{n}^{k_{j}}, u_{n}\right)=\rho\left(z_{n}^{\tau_{k_{j}}}, u_{n}\right) \geq \frac{\delta(\varepsilon)}{2}
$$

by (3.12). From (3.13), we have $|\xi(0)| \leq \delta_{0}$. It follows that

$$
\rho\left(\xi_{n}, u_{n}\right) \rightarrow 0
$$

as $n \rightarrow \infty$ which contradicts (3.15). This completes the proof.

We assume the following:

(H1) $f: \mathbb{Z} \times \mathbb{R}^{d} \rightarrow \mathbb{R}^{d}$ is continuous in $x \in \mathbb{R}^{d}$ for every $n \in \mathbb{Z}$ and is almost periodic in $n \in \mathbb{Z}$ uniformly for $x \in \mathbb{R}^{d}$.

$(\mathrm{H} 2) B: \mathbb{Z} \times \mathbb{Z}^{*} \rightarrow \mathbb{R}^{d}$ is continuous in $x, y \in \mathbb{R}^{d}$ for any $n \in \mathbb{Z}, j \in \mathbb{Z}^{-}$ and is almost periodic in $n \in \mathbb{Z}$ uniformly for $(j, x, y) \in \mathbb{Z}^{*}$. Moreover, for any $\varepsilon>0$ and $\tau>0$, there exists a number $M=M(\varepsilon, \tau)>0$ such that

$$
\sum_{j=-\infty}^{-M}|B(n, j, x(n+j), x(n))|<\varepsilon
$$

for all $n \in \mathbb{Z}$ whenever $|x(j)| \leq \tau, j \in \mathbb{Z}^{-}$.

(H3) Eq. (3.1) has a bounded solution $u(n)$, that is, $|u(n)| \leq c$ for some $c>0$, passing through $\left(0, u_{0}\right), u_{0} \in B S\left(\mathbb{Z}^{-}, \mathbb{R}^{d}\right)$.

Theorem 3.4. For Eq. (3.1) suppose that (H1),(H2), and (H3). Assume that the bounded solution $u(n)$ of $(3.1)$ is $B S$-s.d. $\Omega(f, B)$. Then $u(n)$ is $(K, \rho)$-s.d. $\Omega(f, B)$ if and only if it is $\left((K, \rho), \mathbb{R}^{d}\right)$-s.d. $\Omega(f, B)$.

Proof. $(\Rightarrow)$ It is obvious.

$(\Leftarrow)$ Let $\varepsilon>0,(\tau, \phi) \in \mathbb{Z}^{+} \times B S\left(\mathbb{Z}^{-}, \mathbb{R}^{d}\right)$, and $(g, D) \in \Omega(f, B)$ such that $\phi(s) \in K$ for $s \leq 0, \pi((f, B),(g, D))<\eta$, and $\rho\left(\phi, u_{\tau}\right)<\eta$, where $\eta(\cdot)$ is the one for $\left((K, \rho), \mathbb{R}^{d}\right)$-s.d. $\Omega(f, B)$ of $u(n)$. We show that $\rho\left(x_{n}, u_{n}\right)<\varepsilon$, where $x(n)$ is a solution of the limiting equation of (3.1), passing through $(\tau, \phi)$.

Since $u(n)$ is $B S$-s.d. $\Omega(f, B)$, we have

$$
|x(n)-u(n)|<\varepsilon, n \geq \tau .
$$


Note that

$$
\rho\left(x_{n}, u_{n}\right)=\sum_{j=1}^{\infty} \frac{\rho_{j}\left(x_{n}, u_{n}\right)}{2^{j}\left[1+\rho_{j}\left(x_{n}, u_{n}\right)\right]},
$$

where

$$
\rho_{j}\left(x_{n}, u_{n}\right)=\sup _{-j \leq s \leq 0}\left|x_{n}(s)-u_{n}(s)\right|=\left|x_{n}-u_{n}\right|_{j} .
$$

We estimate $\left|x_{n}-u_{n}\right|_{j}$.

Suppose that $j \leq n-\tau$. Then, by (3.16),

$$
\left|x_{n}-u_{n}\right|_{j}=\sup _{-j \leq s \leq 0}|x(n+s)-u(n+s)|<\varepsilon .
$$

Suppose that $j \geq n-\tau$. From (3.16), we have

$$
\begin{aligned}
\left|x_{n}-u_{n}\right|_{j}= & \max \left\{\sup _{-j \leq s \leq \tau-n}|x(n+s)-u(n+s)|,\right. \\
& \left.\sup _{\tau-n \leq s \leq 0}|x(n+s)-u(n+s)|\right\} \\
\leq & \max \left\{\sup _{-j \leq \theta \leq 0}|\phi(\theta)-u(\tau+\theta)|, \sup _{\tau \leq \theta}|x(\theta)-u(\theta)|\right\} \\
< & \left|\phi-u_{\tau}\right|_{j}+\varepsilon .
\end{aligned}
$$

Thus we obtain

$$
\begin{aligned}
\rho\left(x_{n}, u_{n}\right) & =\sum_{j=1}^{k} \frac{\left|x_{n}-u_{n}\right|_{j}}{\left.2^{j}\left[1+\left|x_{n}-u_{n}\right|_{j}\right)\right]}+\sum_{j=k+1}^{\infty} \frac{\left|x_{n}-u_{n}\right|_{j}}{\left.2^{j}\left[1+\left|x_{n}-u_{n}\right|_{j}\right)\right]} \\
& <\sum_{j=1}^{k} \frac{\varepsilon}{2^{j}(1+\varepsilon)}+\sum_{j=k+1}^{\infty} \frac{\left|\phi-u_{\tau}\right|_{j}+\varepsilon}{2^{j}\left[1+\left|\phi-u_{\tau}\right|_{j}+\varepsilon\right]} \\
& <\varepsilon+\eta .
\end{aligned}
$$

Therefore $u(n)$ is $(K, \rho)$-s.d. $\Omega(f, B)$ with $\delta(\dot{\overline{2}})$. This completes the proof.

Theorem 3.5. Suppose that (H1), (H2), and (H3) for Eq. (3.1). If the bounded solution $u(n)$ of $(3.1)$ is $(K, \rho)$-s.d. $\Omega(f, B)$, then (3.1) has an almost periodic solution.

Proof. In view of [6, Theorem 2] $u(n)$ is asymptotically almost periodic. Then $u(n)$ is almost periodic by [2, Theorem 3.2].

REMARK 3.6. In Theorem 3.2, if $u(n)$ is $(K, \rho)$-totally stable, then (3.1) has an almost periodic solution by [2, Theorem 3.8]. 
REMARK 3.7. For the functional difference equation

$$
x(n+1)=f\left(n, x_{n}\right), n \in \mathbb{Z}^{+},
$$

where $f: \mathbb{Z}^{+} \times B \rightarrow \mathbb{R}^{d}$ and $B$ is the abstract phase space in [6], Hamaya [6] proved that $B S$-s.d. $\Omega(f)$ implies $(K, \rho)$-s.d. $\Omega(f)$. This is the discretization of the result about the functional differential equation

$$
x^{\prime}(t)=h\left(t, x_{t}\right), t \in \mathbb{R}^{+},
$$

where $h: \mathbb{R}^{+} \times B \rightarrow \mathbb{R}^{n}$ in $[8]$.

\section{Acknowledgments}

The authors are thankful to the anonymous referees for their valuable comments to improve this paper.

\section{References}

[1] R. P. Agarwal, Difference Equations and Inequalities, 2nd Edition, Dekker, New York, 2000.

[2] S. K. Choi and N. Koo, Almost periodic solutions of nonlinear discrete Volterra equations with unbounded delay, Adv. Difference. Equ., Vol. 2008, Article ID 692713, 15 pages.

[3] S. K. Choi, Y. H. Goo, D. M. Im, and N. Koo, Total stability in nonlinear discrete Volterra equations with unbounded delay, Abstr. Appl. Anal., Vol. 2009, ID 976369, 13 pages.

[4] Y. Hamaya, Periodic solutions of nonlinear integrodifferential equations, Tohoku Math. J. 41(1989), 105-116.

[5] Y. Hamaya, Stability property for an integrodifferential equation, Differential Integral Equations 6 (1993), 1313-1324.

[6] Y. Hamaya, Existence of an almost periodic solution in a difference equation with infinite delay, J. Difference Equ. Appl. 9 (2003), 227-237.

[7] Y. Hamaya, Stability properties and existence of almost periodic solutions of Volterra difference equations, Discrete Contin. Dyn. Syst. 2009, Dynamical Systems, Differential Equations and Applications, 7th AIMS Conference, suppl., 315-321.

[8] S. Murakami and T. Yoshizawa, Relationships between $B C$-stabilities and $\rho$ stabilities in functional differential equations with infinite delay, Tohoku Math. J. 44 (1992), 45-57.

[9] G. R. Sell, Periodic solutions and asymptotic stability, J. Diff. Equ. 2 (1966), 143-157.

[10] Y. Song and H. Tian, Periodic and almost periodic solutions of nonlinear discrete Volterra equations with unbounded delay, J. Comput. Appl. Math. 205 (2007), 859-870. 
[11] Y. Song, Asymptotically almost periodic solutions of nonlinear Volterra difference equations with unbounded delay, J. Difference Equ. Appl. 14 (2008), 971-986.

[12] Y. Xia and S. S. Cheng, Quasi-uniformly asympototic stability and existence of almost periodic solutions of difference equations in population dynamic systems, J. Difference Equ. Appl. 14 (2008), 59-81.

[13] T. Yoshizawa, Stability Theory and the Existence of Periodic Solutions and Almost Periodic Solutions, Applied Mathematical Sciences 14, Springer-Verlag, New York, 1975.

[14] C. Zhang, Almost Periodic Type Functions and Ergodicity, Science Press, Beijing, China; Kluwer Academic Publishers, Dordrecht, The Netherlands, 2003.

Department of Mathematics Chungnam National University

Daejeon 305-764, Republic of Korea

E-mail: sgchoi@cnu.ac.kr

$* *$

Department of Mathematics

Chungnam National University

Daejeon 305-764, Republic of Korea

$* * *$

Department of Mathematics

Chungnam National University

Daejeon 305-764, Republic of Korea

E-mail: njkoo@cnu.ac.kr

$* * * *$

Department of Mathematics

Chungnam National University

Daejeon 305-764, Republic of Korea

E-mail: cmyun@cnu.ac.kr 